\title{
QUANTITATIVE ASSESSMENT OF APICALLY EXTRUDED DEBRIS AND RETREATABILITY OF TWO SEALERS USING TWO ROTARY RETREATMENT SYSTEMS
}

\author{
Abeer Saba*, Reham Saeed Seyam* and Dalia A. Saba**
}

\begin{abstract}
Objective: Quantitative assessment of apically extruded debris and retreatability of two sealers using two rotary retreatment systems

Methodology: Two NiTi rotary file systems; ProTaper Universal (PT) retreatment files (Dentsply-Maillefer, Ballaigues, Switzerland) and Mtwo R retreatment (MT) rotary files (Sweden and Martina, Padova, Italy) and two sealers; AH Plus (Dentsply International Inc., York, PA, USA) and SmartPaste Bio (DRFP Ltd., Stamford, UK) were used in this study. The root canals of 40 single-rooted human premolar teeth having nearly similar length were prepared. The prepared root canals were obturated with gutta-percha and either AH Plus or Smart Paste Bio sealers ( $n=20 /$ group). Each group was then sub-divided into two subgroups ( $\mathrm{n}=10$ lsubgroup) according to whether the root filling material was removed using either Protaper Universal retreatment or MTwo Retreatment Kit.
\end{abstract}

After retreatment, the apically extruded debris was collected in Eppendorf tubes which were weighed before and after retreatment with an electronic balance. The retreatability of the both sealers was assessed using $\mathrm{CBCT}$ imaging by calculating the volume of the residual filling material post-treatment in the middle and apical thirds of the root canals. Results were statistically analyzed using one-way-analysis-of-variance; $(\mathrm{P}<0.05)$.

Results: Statistically, there was no significant difference in the weight of collected apically extruded debris nor in in the remaining volume of the obturating materials between all groups $(\mathrm{P}=0.849$ and $\mathrm{P}=0.8831)$, respectively.

Conclusions: All investigated retreatment techniques caused debris extrusion and left filling remnants inside the root canals. SmartPaste Bio sealer is as retreatable as AH Plus sealers.

KEYWORDS: AH Plus sealer, apical debris extrusion, CBCT, endodontic retreatment, Protaper retreatment, Mtwo R, Smart Paste Bio sealer.

\footnotetext{
* Assistant Professor of Endodontics, Endodontic Department, Faculty of Dentistry, Cairo University

** Lecturer of Dental Materials Science, Biomaterials Department, Faculty of Dentistry, Cairo University
} 


\section{INTRODUCTION}

Nowadays, preservation of tooth is considered of prime importance to both the dentists and patients. Although endodontic treatment has a long success history, its failure rate has been reported to be 14 to $16 \%$. Treatment alternatives in cases of endodontic treatment failure include either conventional non-surgical (orthograde retreatment) or surgical endodontic retreatment. Non-surgical endodontic retreatment is the treatment of choice with a reported success rate of $74-98 \%$.

The main goal of the non-surgical endodontic retreatment procedures is proper cleaning of the root canal to promote healing of the periapical tissues. Therefore, a successful endodontic retreatment involves complete removal of the root canal filling materials in order to gain access to the apical foramen. This is followed by effective cleaning and shaping, and finally appropriate final refilling of the canal. ${ }^{1-10}$

During retreatment procedures, a common finding is the extrusion of the filling material, necrotic pulp tissues, microorganisms and irrigants into the periapical tissues. Consequently, this might lead to postoperative inflammation, pain, flare-up or even failure of apical healing. It has been reported that almost all techniques whether manual or rotary retreatment techniques are associated with apical extrusion of debris, although being less with rotary instruments. Therefore, one of the factors for a successful retreatment technique involves complete removal of root canal filling material with minimal apical debris extrusion. ${ }^{1-3,5,11}$

Many procedures have been advocated for gutta-percha removal (whether manual, mechanical or ultrasonic), with or without the use of solvents $\mathrm{Ni}$-Ti rotary retreatment systems were found to be both effective and safe with minimal apical debris extrusion in removal of obturation material from the root canal. Mtwo R (retreatment) rotary files (Sweden and Martina, Padova, Italy) and ProTaper
Universal retreatment files (Dentsply-Maillefer, Ballaigues, Switzerland) are two of the NiTi rotary retreatment systems that have been introduced and are specially designed for removing the pre-existing root canal filling materials. The MTwo R system has two instruments, R1-15/.05 and R2- 25/.05, both with $21 \mathrm{~mm}$, which are used at 250 to $300 \mathrm{rpm}$, whereas ProTaper-UR system has three instruments, D1-30/.09-16 mm, D2-25/.08-18 $\mathrm{mm}$, and D3-20/.07-22 mm, which are used at $500 \mathrm{rpm} .^{7,12}$

Another factor to increase the success chances of orthograde retreatment, is the obturating material type which will affect its complete removal from the root canal. The most commonly used obturating material is the gutta percha and sealer. Many sealers have been used in endodontics with AH Plus epoxy resin sealer the golden standard against which other recent sealers are compared. More recently, Bioceramic sealer such as Smartpaste bio have been introduced. Smartpaste bio is a resin based sealant. The manufacturer claims that the addition of bioceramics, gives the sealer excellent dimensional stability and makes it non-resorbable inside the root canal. Smartpaste bio produces calcium hydroxide and hydroxyapatite as byproducts of the setting reaction, rendering the material both anti-bacterial while setting and very biocompatible once set. ${ }^{13-16}$ Yet concerns were raised about ability to retreat canals obturated by bioceramic sealers. In an in vitro study by Hess et al 2011 found that conventional retreatment techniques are not always able to fully remove bioceramic sealers due to their hard mass upon setting. ${ }^{17}$

Therefore, the aim of this study was to quantitatively assess the amount of apically extruded debris using two NiTi rotary (Mtwo R and Protaper retreatment) instrumentation systems. In addition, this study aims to compare the retreatability of two endodontic sealers (smartpaste Bio and AH Plus) using cone beam computed tomography. Therefore, the null hypothesis of the present study that there 
was no difference between two rotary file systems (Protaper and MTwo R) nor between two sealers (AH Plus and Smartpaste Bio) regarding either the amount of apically extruded debris or the amount of remaining obturating material.

\section{MATERIALS AND METHODS}

\section{Materials:}

Two NiTi rotary file systems were used in this study; ProTaper Universal (PT) retreatment files (Dentsply-Maillefer, Ballaigues, Switzerland) and Mtwo R retreatment (MT) rotary files (Sweden and Martina, Padova, Italy).

In addition, two sealers were used; AH Plus (Dentsply International Inc., York, PA, USA) and SmartPaste Bio (DRFP Ltd., Stamford, UK) as shown in Table 1

\section{Methods:}

\section{Specimen selection}

Forty extracted human premolars with single root canals and curvatures $<10$ degrees were selected for this study. The teeth lengths were standardized to approximately $17 \mathrm{~mm}$ by using a diamond disc. The working length (WL) of each canal was determined with the use of \#10 K-file (Dentsply Maillefer, Bellaigues, Switezerland) to 1 $\mathrm{mm}$ short of the apical foramen so that a uniform WL of $16 \mathrm{~mm}$ was achieved for all teeth. The apical root canal diameter was ensured to be not greater than \#20 K-file. ${ }^{1}$

\section{Canal Preparation and Obturation}

Root canal preparations were performed with a modified step-back flare technique. The coronal portion was initially flared with \#2 and \#3 Gates Glidden drills (Mani, Tochigi, Japan). Canals were then prepared with $\mathrm{K}$-files to a master apical file (MAF) \#40 and step back in $1 \mathrm{~mm}$ increment to a file \#60. Patency was maintained by inserting \#20 $\mathrm{K}$-file slightly beyond the apical foramen during recapitulation.

Irrigation with $2 \mathrm{ml}$ of $5.25 \%$ sodium hypochlorite $(\mathrm{NaOCl})$ was done after each instrument. In order to remove the smear layer, $5 \mathrm{ml}$ of $17 \%$ EDTA

TABLE (1) Sealers used in this study

\begin{tabular}{|l|l|l|}
\hline Sealer & Composition & Manufacturer \\
\hline AH Plus & Epoxide paste : Diepoxide & Dentsply International \\
& Calcium tungstate & Inc., York, PA, USA \\
& Zirconium oxide & \\
& Aerosil & \\
& Pigment & \\
& Amine paste: 1-adamantane amine & \\
& N,N'-dibenzyl-5-oxa-nonandiamine-1,9 & \\
& TCD-Diamine & \\
& Calcium tungstate & \\
& Zirconium oxide & \\
& Aerosil & \\
\hline SmartPaste Bio & Silicone oil & Zirconium oxide, tricalcium silicate, dicalcium silicate, calcium hydroxide, \\
& filler & DRFP Ltd., Stamford, UK \\
& and thickening agents & \\
\hline
\end{tabular}


followed by $5 \mathrm{ml}$ of $5.25 \% \mathrm{NaOCl}$ was used. Canals were finally flushed with $10 \mathrm{ml}$ saline solution (Al Mottahedoon Pharma, Egypt) and dried with paper points.

All specimens were randomly divided into four equal groups $(n=10)$ according to the type of sealer and the rotary retreatment files used:

Group 1: to be sealed with AH Plus sealer and retreated with ProTaper Universal retreatment files.

Group 2: to be sealed with AH Plus sealer and retreated with Mtwo $\mathrm{R}$ retreatment files.

Group 3: to be sealed with SmartPaste Bio and retreated with ProTaper Universal retreatment files.

Group 4: to be sealed with SmartPaste Bio and retreated with Mtwo R retreatment files.

Obturation was done using the lateral compaction technique with \#40 gutta-percha (Gapadent, Korea) master cone and \#25 cones as accessories. The access cavities were sealed with Cavit (Dentsply DeTrey, Germany) and teeth were radiographed in buccolingual and mesiodistal directions to confirm the quality of obturation. Specimens were then stored at $37^{\circ} \mathrm{C}$ in an incubator for 30 days to allow complete setting of the sealers.

\section{Retreatment procedure and apically extruded de- bris collection:}

The experimental model used to evaluate debris extrusion was according to that previously described by Kustarci et al 2012; holes were made in the stoppers of Eppendorf tubes and teeth were inserted under pressure through the stoppers. Eppendorf tubes were then fitted into coded glass vials. In no case the inner Eppendorf tube was touched by fingers. Before retreatment, the Eppendorf tubes were weighed with an electronic balance (Scaltec Instruments. GmbH Rudolf-Heiligenstadt, Germany) to $10^{-3}$ precision. Three measurements were taken for each tube and the mean value was recorded..$^{3}$
For all specimens, the first $4-5 \mathrm{~mm}$ of guttapercha was removed from the coronal part of the root with \# 3 Gates-Glidden drills. The retreatment instruments were carried into the canal using electric endodontic motor (X smart, Dentsply, Maillefer, Ballaigues, Switzerland). Speed and torque were set for each instrument according to the manufacturers' instructions. Specimens in each group were retreated as follows:

\section{Group 1 and 3:}

Root fillings were removed using ProTaper Universal retreatment files ( $\mathrm{n}=20$ teeth). The D1, D2, and D3 retreatment instruments were activated by $\mathrm{X}$-Smart ( $2 \mathrm{~N} / \mathrm{cm}$ torque, 500 -rpm speed) and used with a brushing action against the canal walls in a crown-down direction until reaching the working length. D1 worked in the cervical third, D2 in the middle third, and D3 in the entire working length, according to the manufacturer's instructions.

\section{Group 2 and 4:}

Root fillings were removed using Mtwo $\mathrm{R}$ retreatment files ( $n=20$ teeth). The $\mathrm{R} 2$ instrument (size 25/ .05 taper) was activated by the same electric motor adjusted to $2 \mathrm{~N} / \mathrm{cm}$ torque and $300 \mathrm{rpm}$ speed according to the manufacturer's instructions. The file worked with brushstroke movements against the canal walls in a crown-down direction until reaching the WL.

No solvent was used in any of the groups. Progression of the rotary files was performed by applying slight apical pressure and frequently removing the files to inspect the blade and clean the debris from the flutes.

Retreatment was considered complete and that the obturating material was fully removed from the canal when no gutta-percha or sealer could be observed on the retreatment instruments and the canal walls were smooth. In all groups, the canals were finally rinsed with $5 \mathrm{ml}$ saline solution. 
On completion of the retreatment procedure, the Eppendorf tubes were removed from the vials. Apical extrusion of debris was measured by weighing the tube after the retreatment procedures and subtracting the values from the initial weight of the empty Eppendorf tube. Once again, three consecutive measurements were taken and the mean value was recorded. ${ }^{1}$

\section{Retreatability of the two sealers using Cone-Beam Computed Tomography (CBCT) imaging:}

All the teeth were scanned after retreatment by a CBCT scanner (i-CAT; Imaging Sciences International, LLC, Hatfield, PA). CBCT data was imported into mimics software (materialize, Belgium), and the correct orientation of all scans was initially ensured. Then, a certain threshold was assigned to create a mask (according to the optical density) representing remaining gutta percha. The exact threshold was assigned for all the teeth. After creating the threshold the created mask was cropped to one tooth, where manual cropping was mandatory to confine the assigned mask to the root canal lumen, i.e. from the root canal orifice to the apical foramen. The created mask is now confined to root canal space, then the software was set to calculate the volume of the remaining gutta percha based on its threshold value, the reading was recorded and tabulated. Then the ratio of the volume of the residual filling material to the volume of the section is then calculated at both the apical and the middle thirds of each root section as shown in Figure (1). The same procedure was then done for each tooth individually. ${ }^{18-20}$

\section{Statistical analysis}

The results were statistically analyzed using Statistical Package for Social Sciences, Version 21 for Windows (SPSS Inc., an IBM company, USA). Results were presented as means and standard deviations. Values were analyzed using one-way analysis of variance ANOVA and Bonferroni t-test for pairwise comparison to assess significant changes within each group and (independent t-test) to compare the two groups. P-values less than 0.05 were considered statistically significant. For the retreatability test, Multifactor ANOVA was used to statistically analyze the contribution of each factor (Sealer, File type and section) on the remnants with the removal of the effects of all other factors.

\section{RESULTS}

\section{Results of apically extruded debris collection:}

The results of the weight (in grams) of the collected apically extruded debris for the four groups, is shown in Figure (2). Statistically, there was no significant difference in the weight of collected apically extruded debris between all groups $(\mathrm{P}=0.849)$. Comparing the weight of collected apically extruded debris of AH Plus sealer using PT and MT file systems, the mean weight

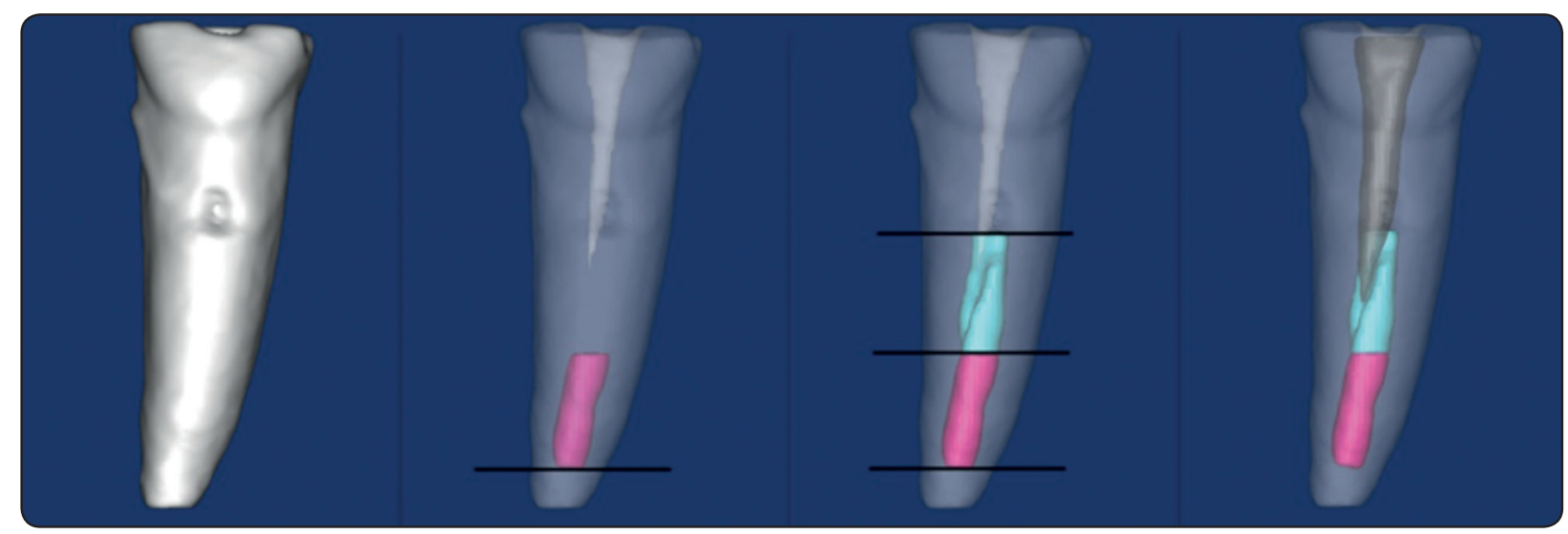

Fig. (1) CBCT reconstruction of representative samples after obturating material removal 
of debris extrusion was $0.00180 \pm 0.00158 \mathrm{~g}$ and $0.00193 \pm 0.000961 \mathrm{~g}$ for the two file systems respectively $(\mathrm{P}=0.864)$. Meanwhile, the weight of collected apically extruded debris of SmartPaste Bio Sealer using PT and MT file systems, the mean weight of debris extrusion was $0.00233 \pm 0.000568$ $\mathrm{g}$ and $0.00203 \pm 0.000935 \mathrm{~g}$ for the two file systems respectively $(\mathrm{P}=0.517)$.

\section{Results of the retreatability of the two sealers:}

The result of the remaining volume of the obturating materials of the four groups, is shown in Figures (3). Statistically, there was no significant difference in the remaining volume of the obturating materials between all groups $(\mathrm{P}=0.8831)$.

The results of Multifactor ANOVA for the contribution of each factor (Sealer, File type and section) on the remnants is measured removing the effects of all other factors. As shown in Figure (4), the sealer-file interaction has a statistically significant effect on the remnants at the $95.0 \%$ confidence level where P-value is less than 0.05 ( $\mathrm{P}=0.0478$ ). Group 1, (AH Plus with PT) showed the statistically highest remaining volume $(26.48 \pm 3.38)$ when compared to group 2 (AH Plus with MT), group 3 (SmartPaste Bio with PT) and group 4 (SmartPaste Bio with MT); 14.93, 13.25 and 15.54, respectively.

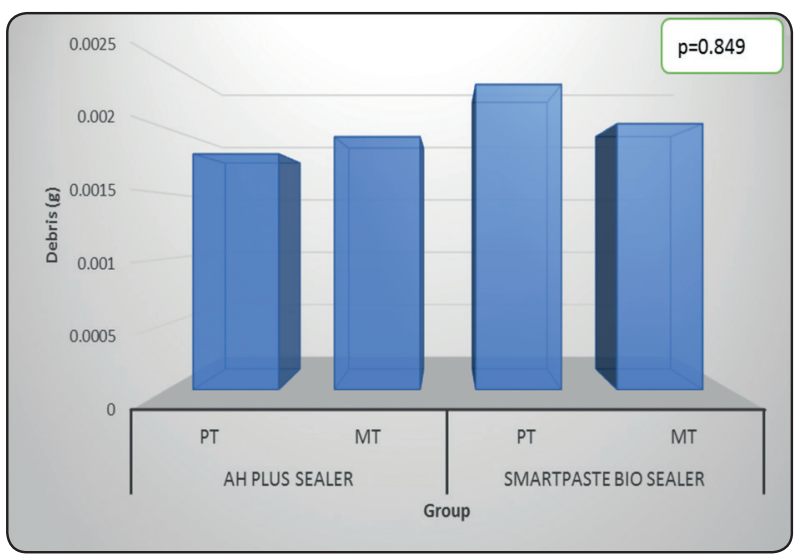

Fig. (2): A bar chart showing mean debris weight (in grams) for the four different groups

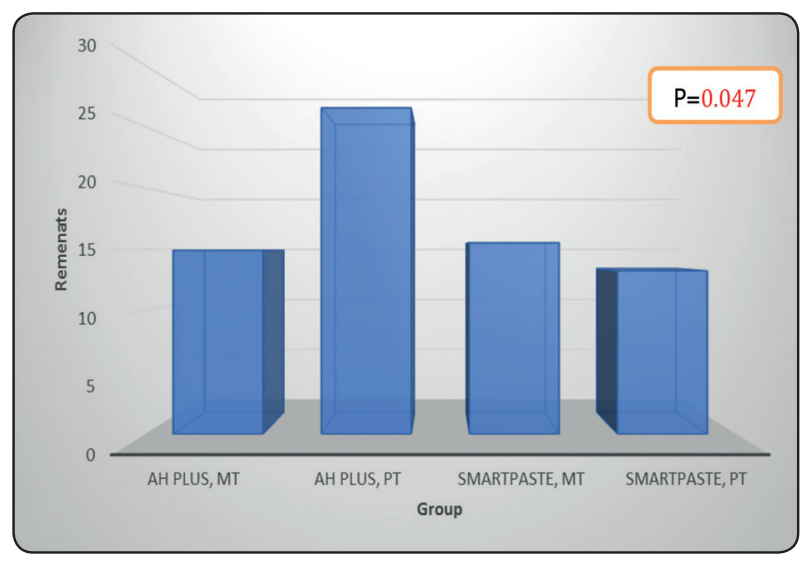

Fig. (3): A bar chart showing least square means of remnants for significant sealer-file type interaction

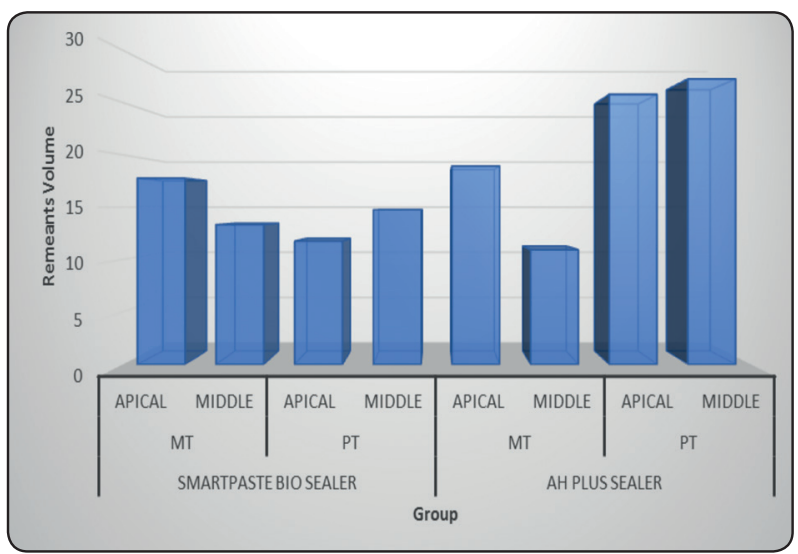

Fig. (4): A bar chart showing mean volume of the sealer remnants for the four different groups

\section{DISCUSSION}

In the present study, several issues were considered during the preparation and retreatment procedures in order to decrease the amount of extruded debris apically and the remaining obturating material within the canal lumen. Decoronation of the teeth was done to ensure the standardization of all roots and to allow easy access to the root canals as it eliminates the effect of crown anatomy. In addition, the working length was confined to $1 \mathrm{~mm}$ shorter than the apical foramen as it was stated that this helps to decrease the amount of extruded debris apically. Crown-down technique was used during the retreatment procedures in the current study. Many 
studies have shown that crown-down technique resulted in lesser extrusion of debris when compared to step-back technique. Moreover, saline was used as the irrigation solution during the retreatment procedures to avoid any possible crystallization of $\mathrm{NaOCl}$ and hence greater amount of extruded debris and remaining filling material. No solvent was used with Ni-Ti retreatment instruments as they plasticize gutta-percha through the heat generated by friction, allowing them to reach the working length easily and quickly._Moreover, ProTaper and Mtwo R retreatment files, were selected in this study as they have cutting tips may help in easier penetration into the filling material. ${ }^{1-3,5,8,11,12,21}$

The most commonly used methods to assess the volume of the remaining obturating materials involve longitudinal sectioning of the teeth followed by digital imaging or scanning electron microscope analysis. However, these methods are 2 dimensional methods and they are semi-quantitative. Recently, micro CT has been introduced in endodontics as a tool to evaluate the amount of remaining filling material. However, micro CT is not suitable for clinical use. It has been established that high resolution $\mathrm{CBCT}$ is suggested as an alternative to micro CT. ${ }^{22}$

The results of the present study revealed that all investigated four groups presented extrusion of debris apically and showed a remaining obturating material during the CBCT imaging. These findings is in agreement with other studies which emphasis the fact that it is impossible to neither retreat a root canal without apical extrusion of debris nor complete removal of the obturating material regardless of the rotary file system used or the type of sealer used. In addition, the results showed no significant difference between either rotary file systems (ProTaper retreatment or MTwo R) or between either sealers (AH Plus or SmartPaste Bio) regarding either apically extruded debris or the amount of remaining obturating materials. Therefore, the null hypothesis of the present study was accepted.
On the other hand, multifactorial ANOVA revealed that ProTaper retreatment file system combined with AH Plus sealer had significantly greater remaining volume of the obturating material when compared with the other investigated groups $(\mathrm{P}=0.0478)$. This might be attributed to the fact that ProTaper retreatment file system has a convex triangular cross-section which might reduce the area of contact between the instruments and the canal wall. In addition, the centering tendency of the D series files could be another explanation for the more remaining obturating material. ${ }^{5,21}$ Another reason that the last apical retreatment protaper instrument is D3 with \# 20 at the tip which is too small to completely clean apical part of the canal when compared to Mtwo retreatment instrument with \# 25 at the tip. ${ }^{23}$

In contrast, in the Mtwo file, the distance between the cutting edges (pitch) is increased from the tip of the instrument to the handle. The depth of the space designed for dentin removal is increased behind the blades, which provides the largest space for dentin removal and leads to more efficient gutta-percha and sealer removal. This goes in agreement with the study by Schäfer and Oitzinger which stated that the Mtwo R files have a small core diameter, great chip removal capacity and great chip space that can result in great cutting ability. ${ }^{7,21}$

Regarding the retrievability of root canal sealer, sealer properties such as adhesion to dentin and gutta-percha, degree of penetration into the dentinal tubules, film thickness, dimensional change, as well as solubility are of prime importance. AH plus sealer is an epoxy-resin based sealer, with good adhesion to dentinal walls as it is known to penetrate up to 10$80 \mu \mathrm{m}$ into the dentinal tubules. Therefore, it was difficult to be removed during retreatment. ${ }^{24}$ This was in consistent with the results of the study by Kim et al 2015, which showed a significantly higher percentage in sealer penetration depth in the $\mathrm{AH}$ Plus group when compared to a bioceramic sealer. The study had concluded that this might have been caused by the relatively higher fluidity of AH Plus 
sealer. In addition, these findings are in accordance with previous studies that confirmed that AH Plus sealer, were also difficult to be completely removed as it had penetrated slightly deeper into the dentin than other bioceramic sealer. ${ }^{25}$

However, it has to be mentioned that the incomplete removal of obturating material cannot be considered a retreatment failure. It has been stated that to completely remove the penetrating sealer material, one would have to remove up to $40 \%-60 \%$ of additional root dentin. ${ }^{25}$

\section{CONCLUSIONS}

Within the limitation of this study, the following were concluded:

1. All investigated retreatment techniques caused debris extrusion and left filling remnants inside the root canals.

2. SmartPaste Bio sealer is as retreatable as $\mathrm{AH}$ Plus sealers.

\section{RECOMMENDATIONS}

Caution should be taken on applying the results of this in vitro study on clinical situations. In the apically extruded debris collection test, the teeth apex were suspended in air, whereas in vivo the apex would be enclosed by granulomatous or periradicular tissues, which may act as a natural barrier limiting apical extrusion of debris. ${ }^{2,3}$

Furthermore, our current study was carried out on teeth with straight root canals; therefore, our findings cannot be directly correlated to teeth with curved root canals. Therefore, further studies need to be carried out to investigate the efficiency of rotary nickel titanium retreatment instruments in teeth with curved root canals. ${ }^{8}$

Because this study aimed at comparing the efficacy of retreatment instruments for removing obturating material to the working length, the canals were not reinstrumented with larger files as recommended by other studies. The final tip sizes in each retreatment system were D3 (size 20) and Mtwo $\mathrm{R}$ (size 25) which might have led to the presence of apical filling debris. Thus further studies need to evaluate the effect of canal reinstrumentation with larger sized instruments after retreatment procedure to obtain better apical cleaning.

\section{REFERENCES}

1. Uezu MKN, Britto MLB, Nabeshima CK, Pallotta RC. Comparison of debris extruded apically and working time used by ProTaper Universal rotary and ProTaper retreatment system during gutta-percha removal. J Appl Oral Sci. 2010;18(6):542-545. doi:10.1590/S167877572010000600002 .

2. Huang X, Ling J, Wei X, Gu L. Quantitative Evaluation of Debris Extruded Apically by Using ProTaper Universal Tulsa Rotary System in Endodontic Retreatment. J Endod. 2007;33(9):1102-1105. doi:10.1016/j.joen.2007.05.019.

3. Kustarci A, Altunbas D, Akpinar KE. Comparative study of apically extruded debris using one manual and two rotary instrumentation techniques for endodontic retreatment. J Dent Sci. 2012;7(1):1-6. doi:10.1016/j.jds.2011.09.011.

4. Eduardo C, Miranda DA, Souza-junior EJ, Baggio FH. Evaluation of surface roughness and color stability of direct resin composites after different polishing protocols. Int J Dent Clin. 2011;3(3):4-7.

5. Topçuołlu HS, Akti A, Tuncay Ö, Dinçer AN, Düzgün S, Topçuołlu G. Evaluation of debris extruded apically during the removal of root canal filling material using ProTaper, $\mathrm{D}-\mathrm{RaCe}$, and R-Endo rotary nickel-titanium retreatment instruments and hand files. J Endod. 2014;40(12):20662069. doi:10.1016/j.joen.2014.09.004.

6. Arora C, Bahri R, Mittal N. Comparative evaluation of debris extruded apically by using, Protaper retreatment file, $\mathrm{K} 3$ file and $\mathrm{H}$-file with solvent in endodontic retreatment. Saudi End J. 2012;2(3):136-141. doi:10.4103/16585984.112706.

7. Akhavan H, Azdadi YK, Azimi S, Dadresanfar B, Ahmadi A. Comparing the efficacy of Mtwo and D-RaCe retreatment systems in removing residual gutta-percha and sealer in the root canal. Iran Endod J. 2012;7(3):122-126.

8. Joseph M, Ahlawat J, Malhotra A, Rao M, Sharma A, Talwar S. In vitro evaluation of efficacy of different rotary instrument systems for gutta percha removal during 
root canal retreatment. J Clin Exp Dent. 2016;8(4):0-0. doi: $10.4317 /$ jced.52488.

9. Garg A, Nagpal A, Shetty S, Kumar S, Singh KK, Garg A. Comparison of time required by D-RaCe, R-Endo and Mtwo instruments for retreatment: An in vitro study. J Clin Diagnostic Res. 2015;9(2):ZC47-ZC49. doi:10.7860/ JCDR/2015/11100.5596.

10. Uzunoglu E, Yilmaz Z, Sungur D, Altundasar E. Retreatability of Root Canals Obturated Using Retreatability of Root Canals Obturated Using Gutta-Percha. 2015;10(April):93-98.

11. Dincer AN, Er O, Canakci BC. Evaluation of apically extruded debris during root canal retreatment with several NiTi systems. Int Endod J. 2015;48(12):1194-1198. doi:10.1111/iej.12425.

12. Mollo A, Botti G, Prinicipi Goldoni N, Efficacy of two NiTi systems and hand files for removing gutta-percha from root canals. Int Endod J. 2012;45(1):1-6. doi:10.1111/ j.1365-2591.2011.01932.x.

13. Pathivada L, Munagala K, Dang A. Smartseal: New Age Obturation. Ann Dent Spec 2013. 2013;1(1):13-15.

14. Badami V, Ahuja B. Biosmart materials: Breaking new ground in dentistry. Sci World J. 2014; 2014. doi: $10.1155 / 2014 / 986912$.

15. Bueno Cre, Valentim D, Marques V., Biocompatibility and biomineralization assessment of bioceramic-, epoxy-, and calcium hydroxide-based sealers. Braz Oral Res. 2016; 30(1):1-9. doi:10.1590/1807-3107BOR-2016.vol30.0081.

16. Celikten B, Uzuntas CF, Gulsahi K. Resistance to fracture of dental roots obturated with different materials. Biomed Res Int. 2015;2015. doi:10.1155/2015/591031.

17. Hess D, Solomon E, Spears R, He J. Retreatability of a bioceramic root canal sealing material. J Endod. 2011;37(11):1547-1549. doi:10.1016/j.joen.2011.08.016.

18. Rödig T, Hausdörfer T, Konietschke F, Dullin C, Hahn W, Hülsmann M. Efficacy of D-RaCe and ProTaper Universal
Retreatment NiTi instruments and hand files in removing gutta-percha from curved root canals - a micro-computed tomography study. Int Endod J. 2012;45(6):580-589. doi:10.1111/j.1365-2591.2012.02014.x.

19. Sağlam BC, Türker MM, Türker SA, Koçak S. Efficacy of different solvents in removing gutta-percha from curved root canals: A micro-computed tomography study. Aust Endod J. 2014;40(2):76-80. doi:10.1111/aej.12041.

20. La SH, Jung DH, Kim EC, Min KS. Identification of Independent Middle Mesial Canal in Mandibular First Molar Using Cone-Beam Computed Tomography Imaging. J Endod. 2010;36(3):542-545. doi:10.1016/j. joen. 2009.11.008.

21. Dadresanfar B, Mehrvarzfar P, Saghiri MA, Ghafari S, Khalilak Z, Vatanpour M. Efficacy of two rotary systems in removing guttapercha and sealer from the root canal walls. Iran Endod J. 2011;6(2):69-73.

22. Neelakantan P, Grotra D, Sharma S. Retreatability of 2 mineral trioxide aggregate-based root canal sealers: A cone-beam computed tomography analysis. J Endod. 2013;39(7):893-896. doi:10.1016/j.joen.2013.04.022.

23. Marfisi K, Mercade M, Plotino G, Duran-Sindreu F, Bueno R, Roig M. Efficacy of three different rotary files to remove gutta-percha and Resilon from root canals. Int Endod J. 2010;43(11):1022-1028. doi:10.1111/j.13652591.2010.01758.x.

24. Kanaparthy A, Kanaparthy R. The comparative efficacy of different files in the removal of different sealers in simulated root canal retreatment - An in-vitro study. J Clin Diagnostic Res. 2016;10(5):ZC130-ZC133. doi:10.7860/ JCDR/2016/17731.7845.

25. Kim H, Kim E, Lee SJ, Shin SJ. Comparisons of the Retreatment Efficacy of Calcium Silicate and Epoxy Resin-based Sealers and Residual Sealer in Dentinal Tubules. J Endod. 2015;41(12):2025-2030. doi:10.1016/j. joen.2015.08.030. 